\title{
Jet Quenching and Gluon to Hadron Fragmentation Function in Non-Equilibrium QCD at RHIC and LHC
}

\author{
Gouranga C Nayak ${ }^{1}$ \\ 1 22 West Fourth Street \#1, Lewistown, Pennsylvania 17044, USA
}

\begin{abstract}
Theoretical understanding of the observed jet quenching measurements at RHIC and LHC is challenging in QCD because it requires understanding of parton to hadron fragmentation function in non-equilibrium QCD. In this paper, by using closed-time path integral formalism, we derive the gauge invariant definition of the gluon to hadron fragmentation function in non-equilibrium QCD which is consistent with factorization theorem in non-equilibrium QCD from first principles.
\end{abstract}

PACS numbers: 12.38.Mh, 12.38.Aw, 12.38.Lg, 13.87.Fh 


\section{INTRODUCTION}

During the early stage of the evolution of the universe, just after $\sim 10^{-12}$ seconds of the big-bang, the universe was filled with a state of matter known as quark-gluon plasma. This early stage of the universe is known as the quark epoch. The temperature of the quark-gluon plasma is very high $\gtrsim 3.2 \times 10^{12} \mathrm{~K}(\gtrsim 200 \mathrm{MeV})$ which is about million times larger than the temperature of the sun $\left(\sim 1.56 \times 10^{7} \mathrm{~K}\right)$. Besides black holes, the quark-gluon plasma is much denser than all the forms of the matter we know so far such as neutron stars etc.. Hence it will be a huge step for us if we can recreate this early universe scenario in the laboratory, $i$. e., if we can produce quark-gluon plasma in the laboratory. At present, the RHIC and LHC heavy-ion colliders are the best facilities to create quark-gluon plasma in the laboratory.

RHIC (relativistic heavy ion collider) at BNL collides two gold nuclei at $\sqrt{s}=200 \mathrm{GeV}$ and the LHC (large hadron collider) at CERN (in its first run) collides two lead nuclei at $\sqrt{s}$ $=2.76 \mathrm{TeV}$. Since the total energy at RHIC and LHC are very high $\sim 40 \mathrm{TeV}$ and $\sim 574$ $\mathrm{TeV}$ respectively which are deposited in small volume at the initial time, the initial energy density might have been much higher than the equivalent temperature of $\sim 200 \mathrm{MeV}$ to produce quark-gluon plasma at RHIC and LHC. In the second run at the LHC in the $\mathrm{PbPb}$ collisions at $\sqrt{s}=5.5 \mathrm{TeV}$ the total energy $\sim 1150 \mathrm{TeV}$ will even create much higher initial energy density.

However, the main problem at RHIC and LHC is whether the quark-gluon plasma is thermalized or not. In case of a non-thermalized quark-gluon plasma at RHIC and LHC the notion of temperature does not exist and hence the finite temperature lattice QCD studies become inapplicable at RHIC and LHC. The question of non-equilibrium quarkgluon plasma arises because the two nuclei at RHIC and LHC travel almost at speed of light. This means the partons inside the two nuclei just before the collision at RHIC and LHC carry very high longitudinal momentum and very small transverse momentum creating momentum anisotropy after the nuclear collision. In order for these anisotropic partons to form thermalized quark-gluon plasma many more secondary partonic collisions over a large period of time is required. However, the typical hadronization time in QCD is very small $\left(\sim 10^{-24}\right.$ seconds) after which the partons become hadrons. Hence there may not be enough time for these anisotropic partons to thermalize before hadronization takes place at RHIC 
and LHC. Hence it is necessary to study nonequilibrium-nonperturbative QCD at RHIC and LHC which, of course, is a difficult problem.

It should be remembered that the quark-gluon plasma can not be directly detected at RHIC and LHC because quarks and gluons are not directly observed. The primary indirect signatures of quark-gluon plasma detection are, 1) $j / \psi$ suppression [1] 2) dilepton and direct photon production [2], 3) strangeness enhancement [3] and, 4) jet quenching [4, 5] etc.. We will focus on the jet fragmentation in non-equilibrium QCD in this paper.

Non-equilibrium quantum field theory can be studied by using closed-time path (CTP) integral formalism [6, 7]. Hence in order to study nonequilibrium-nonperturbative QCD it is necessary to use path integral formulation of non-equilibrium QCD by using CTP formalism. It should be mentioned, however, that implementing CTP in non-equilibrium at RHIC and LHC is a very difficult problem, especially due to the presence of gluons in non-equilibrium and hadronization etc. Even the calculation of one-loop gluon self energy in non-equilibrium in covariant gauge which is derived in [8, 9] becomes much more tedious than the calculation of one loop QCD diagram in vacuum due to the presence of additional closed time path indices in non-equilibrium.

Recently, by using Schwinger-Keldysh closed-time path integral formalism, we have derived the gauge invariant definition of the quark to hadron fragmentation function in nonequilibrium QCD [10, 11]. In this paper we will extend this study to derive the gauge invariant definition of the gluon to hadron fragmentation function in non-equilibrium QCD which is obtained from the single gluon in-state $\mid g>$ in non-equilibrium QCD.

The paper is organized as follows. In section II we present the main results and discuss the novel features. In section III we give a brief description of closed-time path integral formalism in non-equilibrium QCD. We implement closed-time path integral formalism in non-equilibrium QCD to derive the definition of the gauge non-invariant gluon to hadron fragmentation function in non-equilibrium QCD in section IV. In section V we prove factorization of infrared divergences in non-equilibrium QCD. In section VI we derive the definition of the gauge invariant gluon to hadron fragmentation function in non-equilibrium QCD. Section VII contains conclusions. 


\section{MAIN RESULTS AND NOVEL FEATURES}

Before presenting the technical details of the derivation of the gluon to hadron fragmentation function in non-equilibrium QCD we will first summarize the main result and its novel features in this section. The readers who are not interested in the technical details can skip the rest of the paper.

\section{A. Main Results}

For the gluon with arbitrary non-equilibrium (non-isotropic) distribution function $f_{g}(\vec{k})$ at initial time $t_{0}$, we find that the gauge invariant definition of the gluon to hadron fragmentation function in non-equilibrium QCD which is obtained from the single gluon in-state $\mid g>$ and is consistent with factorization theorem in non-equilibrium QCD is given by

$$
\begin{aligned}
& D_{H / g}\left(z, P_{T}\right)=\frac{k^{+}}{16 z\left[1+f_{g}\left(k^{+}, k_{T}\right)\right]} \int d x^{-} \frac{d^{d-2} x_{T}}{(2 \pi)^{d-1}} e^{i k^{+} x^{-}+i P_{T} \cdot x_{T} / z} \\
& <i n\left|Q^{\mu a}\left(x^{-}, x_{T}\right) \Phi\left[x^{-}, x_{T}\right] a_{H}^{\dagger}\left(P^{+}, 0_{T}\right) a_{H}\left(P^{+}, 0_{T}\right) \Phi[0] Q_{\mu}^{a}(0)\right| i n>
\end{aligned}
$$

which is valid in covariant gauge, in light-cone gauge, in general axial gauges, in general non-covariant gauges and in general Coulomb gauge etc. where $Q^{\mu a}(x)$ is the (quantum) gluon field which fragments to hadron and $\mid i n>$ is the initial state of the non-equilibrium QCD medium in the Schwinger-Keldysh in - in closed-time path formalism and the path ordered exponential

$$
\Phi_{a b}[x]=\left[\mathcal{P} \exp \left[-i g \int_{0}^{\infty} d \lambda l \cdot A^{c}(x+l \lambda) T^{(A) c}\right]\right]_{a b}, \quad T_{a b}^{(A) c}=-i f^{c a b}
$$

is the non-abelian phase or non-abelian gauge link in the adjoint representation of $\mathrm{SU}(3)$ where $l^{\mu}$ is the light-like four-velocity and $A^{\mu c}(x)$ is the $\mathrm{SU}(3)$ pure gauge background field with $a, b, c=1,2, \ldots, 8$. The definition of the gluon to hadron fragmentation function in non-equilibrium QCD in eq. (1) is gauge invariant with respect to the gauge transformation

$$
T^{c} A_{\mu}^{c}(x)=U(x) T^{c} A_{\mu}^{c}(x) U^{-1}(x)+\frac{1}{i g}\left[\partial_{\mu} U(x)\right] U^{-1}(x)
$$

along with the homogeneous transformation $[12-15]$

$$
T^{c} Q_{\mu}^{\prime c}(x)=U(x) T^{c} Q_{\mu}^{c}(x) U^{-1}(x)
$$


where

$$
U(x)=e^{i g T^{c} \omega^{c}(x)}
$$

The special case $f_{g}(\vec{k})=\frac{1}{e^{\frac{k_{0}}{T}}-1}$ corresponds to the finite temperature QCD in equilibrium.

We will present a derivation of eq. (1) in this paper.

\section{B. Novel and Qualitatively Interesting Features}

Let us describe the novel features of the definition of the gluon to hadron fragmentation function in non-equilibrium QCD obtained in eq. (1).

Similar to DGLAP evolution equation [16] in pp collisions we will follow the DGLAP-like evolution equation procedure in AA collisions at RHIC and LHC where the inclusive hadron production cross section is given by the factorized formula in non-equilibrium QCD

$$
\frac{d \sigma_{A A}^{H}}{d y d p_{T}^{2}}=\sum_{i} \int \frac{d z}{z^{2}} \frac{d \hat{\sigma}_{i}}{d y d p_{T i}^{2}} D_{H / i}(z, Q)
$$

where $\frac{d \hat{\sigma}_{i}}{d y d p_{T i}^{2}}$ is the partonic level differential cross section in non-equilibrium QCD and $D_{H / i}(z, Q)$ is the fragmentation function in non-equilibrium QCD. The $Q^{2}$ evolution of the fragmentation function $D_{H / i}(z, Q)$ in non-equilibrium QCD obeys the generalized DGLAPlike evolution equation in non-equilibrium QCD

$$
\frac{d D_{H / i}(z, Q)}{d l n Q}=\frac{\alpha_{s}(Q)}{\pi} \sum_{j} \int_{z}^{1} \frac{d z^{\prime}}{z^{\prime}} P_{i \rightarrow j k}\left(z^{\prime}, Q\right) D_{H / j}\left(\frac{z}{z^{\prime}}, Q\right)
$$

where $P_{i \rightarrow j k}\left(z^{\prime}, Q\right)$ is the splitting function in non-equilibrium QCD [17]. In the above equations the indices $i, j, k=q, \bar{q}, g$.

Note that one of the crucial ingredient to derive DGLAP evolution equation is the factorization theorem [16]. Hence similar to DGLAP equation in QCD in vacuum the DGLAP-like equation in non-equilibrium QCD in eq. (17) is consistent with the factorization theorem in non-equilibrium QCD (see also eq. (6) and appendix A). This implies that the partonic level differential cross section $\frac{d \hat{\sigma}_{i}}{d y d p_{T i}^{2}}$ in eq. (6) in non-equilibrium QCD and the fragmentation function $D_{H / i}(z, Q)$ in eq. (6) in non-equilibrium QCD are studied by using $\mid$ in $>$ for the ground state in non-equilibrium QCD instead of the usual vacuum state $\mid 0>$ in QCD. It should be remembered that the ground state $\mid$ in $>$ in non-equilibrium QCD contains both the vacuum part and the medium part. For example, when the distribution function 
$f(\vec{k})=0$ then $\mid$ in $>$ becomes $\mid 0>$ and one reproduces all the equations and quantities of the QCD in vacuum.

$[8,[9]$

The leading order perturbative gluon propagator in non-equilibrium QCD is given by

$$
G^{\mu \nu}(k)_{r s}=-i\left[g^{\mu \nu}+(\alpha-1) \frac{k^{\mu} k^{\nu}}{k^{2}}\right] G_{r s}^{\mathrm{vac}}(k)-i T^{\mu \nu} G_{r s}^{\mathrm{med}}(k)
$$

which contains the vacuum propagator $G_{r s}^{\mathrm{vac}}(k)$ plus the medium propagator $G_{r s}^{\text {med }}(k)$ where

$$
G_{r s}^{\mathrm{vac}}(k)=\left(\begin{array}{cc}
\frac{1}{k^{2}+i \epsilon} & -2 \pi \delta\left(k^{2}\right) \theta\left(-k_{0}\right) \\
-2 \pi \delta\left(k^{2}\right) \theta\left(k_{0}\right) & -\frac{1}{k^{2}-i \epsilon}
\end{array}\right)
$$

and

$$
G_{r s}^{\text {med }}(k)=2 \pi \delta\left(k^{2}\right) f_{g}(\vec{k})\left(\begin{array}{cc}
1 & 1 \\
1 & 1
\end{array}\right)
$$

In the above equations the closed-time indices $r, s=+,-$ correspond to upper and lower time branch in the closed-time path formalism.

However, since the gluon fragmentation function is a non-perturbative quantity in QCD it is not possible to decompose the gluon fragmentation function into the vacuum part and the medium part (see eq. (10)). Hence one finds that to be consistent with factorization theorem in non-equilibrium QCD, the fragmentation function in QCD in vacuum can not be used in the DGLAP-like equation in non-equilibrium QCD in eq. (17) to study hadron production from quark-gluon plasma. The fragmentation function in non-equilibrium QCD should be used in DGLAP-like equation in non-equilibrium QCD in eq. (7) to study hadron production from quark-gluon plasma by using factorized formula as given by eq. (6) where $\frac{d \hat{\sigma}_{i}}{d y d p_{T i}^{2}}$ is the partonic level differential cross section in non-equilibrium QCD and $D_{H / i}(z, Q)$ is the fragmentation function in non-equilibrium QCD.

\section{NON-EQUILIBRIUM QCD USING CLOSED-TIME PATH INTEGRAL FOR- MALISM}

When a system is in non-equilibrium its asymptotic future is quite different from its initial preparation in the remote past. Hence unlike zero-temperature field theory or finite temperature field theory in equilibrium there is no simple relation between asymptotic future 
state and initial state in the remote past. For this reason the generic non-equilibrium quantum field theory is very non-trivial. In order to avoid the asymptotic future state and to deal with only initial state in the remote past one introduces two time branches in the closed-time path (CTP) formalism [6, 7].

The gluon to hadron fragmentation function in non-equilibrium QCD in eq. (11) is a non-perturbative quantity. Hence it is necessary to use path integral formulation of nonequilibrium QCD in CTP formalism to study its properties. Before going to gluons in non-equilibrium QCD let us consider the scalar gluons first in the CTP formalism in nonequilibrium in the path integral formulation. The generating functional in scalar field theory in non-equilibrium is given by

$Z\left[\rho, J_{+}, J_{-}\right]=\int\left[d \phi_{+}\right]\left[d \phi_{-}\right] \exp \left[i \int d^{4} x\left[\mathcal{L}\left[\phi_{+}\right]-\mathcal{L}\left[\phi_{-}\right]+J_{+} \phi_{+}-J_{-} \phi_{-}\right]\right]<\phi_{+}, 0|\rho| 0, \phi_{-}>$

where $\rho$ is the initial density of states in non-equilibrium, $\mathcal{L}[\phi]$ is the full lagrangian density in scaler field theory and $\mid \phi_{ \pm}, 0>$ is the quantum state corresponding to the field configuration $\phi_{ \pm}(\vec{x}, t=0)$ at the initial time. The subscript + refers to closed-time path index in the positive time contour and the subscript - refers to closed-time path index in the negative time contour in the closed-time path formalism [6, 7].

Since there are two time branches,+- in the closed-time path formalism there are two external sources $J_{+}, J_{-}$corresponding to these two different time branches. Hence one finds that there are four green's functions in non-equilibrium which are given by

$$
\begin{aligned}
& G_{++}\left(x, x^{\prime}\right)=\frac{\delta Z\left[J_{+}, J_{-}, \rho\right]}{i^{2} \delta J_{+}(x) J_{+}\left(x^{\prime}\right)}=<T \phi(x) \phi\left(x^{\prime}\right)>=<i n\left|T \phi(x) \phi\left(x^{\prime}\right)\right| i n> \\
& G_{--}\left(x, x^{\prime}\right)=\frac{\delta Z\left[J_{+}, J_{-}, \rho\right]}{(-i)^{2} \delta J_{-}(x) J_{-}\left(x^{\prime}\right)}=<\bar{T} \phi(x) \phi\left(x^{\prime}\right)>=<i n\left|\bar{T} \phi(x) \phi\left(x^{\prime}\right)\right| i n> \\
& G_{+-}\left(x, x^{\prime}\right)=\frac{\delta Z\left[J_{+}, J_{-}, \rho\right]}{-i^{2} \delta J_{+}(x) J_{-}\left(x^{\prime}\right)}=<\phi\left(x^{\prime}\right) \phi(x)>=<i n\left|\phi\left(x^{\prime}\right) \phi(x)\right| i n> \\
& G_{-+}\left(x, x^{\prime}\right)=\frac{\delta Z\left[J_{+}, J_{-}, \rho\right]}{-i^{2} \delta J_{-}(x) J_{+}\left(x^{\prime}\right)}=<\phi(x) \phi\left(x^{\prime}\right)>=<i n\left|\phi(x) \phi\left(x^{\prime}\right)\right| i n>
\end{aligned}
$$

where $\mid$ in $>$ is the initial state in non-equilibrium quantum field theory at the initial time $t=t_{i n}=0$. Note that due to the presence of the medium the initial state $|i n\rangle$ in nonequilibrium quantum field theory is different from the vacuum state $\mid 0>$ in the quantum field theory in vacuum. 
In order to extend scalar gluon to gluon in non-equilibrium QCD we need to add gauge fixing term in the lagrangian density which results in the appearance of the Faddeev-Popov (F-P) determinant in the generating functional which can be expressed in terms of path integral over the ghost fields [18]. However, we will directly work with the Faddeev-Popov (F-P) determinant in this paper and we will work in the frozen ghost formalism [8, 9] for the medium part at the initial time $t=t_{i n}=0$. Hence it is now straightforward to extend the generating functional of the scalar gluon in non-equilibrium in eq. (11) to gluon in non-equilibrium QCD.

Extending the generating functional of the scalar gluon in non-equilibrium in eq. (11) to gluon in non-equilibrium $\mathrm{QCD}$ we find that the generating functional in non-equilibrium QCD in the path integral formulation is given by [8, 9]

$$
\begin{aligned}
& Z\left[\rho, J_{+}, J_{-}, \eta_{+}, \eta_{-}, \bar{\eta}_{+}, \bar{\eta}_{-}\right]=\int\left[d Q_{+}\right]\left[d Q_{-}\right]\left[d \bar{\psi}_{+}\right]\left[d \bar{\psi}_{-}\right]\left[d \psi_{+}\right]\left[d \psi_{-}\right] \operatorname{det}\left(\frac{\delta \partial_{\mu} Q_{+}^{\mu c}}{\delta \omega_{+}^{d}}\right) \operatorname{det}\left(\frac{\delta \partial_{\mu} Q_{-}^{\mu c}}{\delta \omega_{-}^{d}}\right) \\
& \exp \left[i \int d ^ { 4 } x \left[-\frac{1}{4}\left(F_{\mu \nu}^{c 2}\left[Q_{+}\right]-F_{\mu \nu}^{c 2}\left[Q_{-}\right]\right)-\frac{1}{2 \alpha}\left(\partial_{\mu} Q_{+}^{\mu c}\right)^{2}+\frac{1}{2 \alpha}\left(\partial_{\mu} Q_{-}^{\mu c}\right)^{2}+\bar{\psi}_{+} D\left[Q_{+}\right] \psi_{+}\right.\right. \\
& \left.\left.-\bar{\psi}_{-} D\left[Q_{-}\right] \psi_{-}+J_{+} \cdot Q_{+}-J_{-} \cdot Q_{-}+\bar{\eta}_{+} \cdot \psi_{+}-\bar{\eta}_{-} \cdot \psi_{-}+\bar{\psi}_{+} \cdot \eta_{+}-\bar{\psi}_{-} \cdot \eta_{-}\right]\right] \\
& \times<Q_{+}, \psi_{+}, \bar{\psi}_{+}, 0|\rho| 0, \bar{\psi}_{-}, \psi_{-}, Q_{-}>
\end{aligned}
$$

where $\rho$ is the initial density of state in non-equilibrium $\mathrm{QCD}$, the state $\mid Q^{ \pm}, \psi^{ \pm}, \bar{\psi}^{ \pm}, 0>$ corresponds to the field configurations $Q_{\mu}^{c}\left(\vec{x}, t=t_{\text {in }}=0\right), \psi\left(\vec{x}, t=t_{\text {in }}=0\right), \bar{\psi}\left(\vec{x}, t=t_{\text {in }}=0\right)$, the $J^{\mu c}(x)$ is the external source for the quantum gluon field $Q^{\mu c}(x)$, the $\bar{\eta}_{i}(x)$ is the external source for the Dirac field $\psi_{i}(x)$ of the quark, $\alpha$ is the gauge fixing parameter, $\operatorname{det}\left(\frac{\delta\left(\partial_{\mu} Q_{ \pm}^{\mu b}\right)}{\delta \omega_{ \pm}^{c}}\right)$ is the Faddeev-Popov (F-P) determinant and

$$
\begin{aligned}
& F_{\mu \nu}^{c}\left[Q_{ \pm}\right]=\partial_{\mu} Q_{\nu \pm}^{c}(x)-\partial_{\nu} Q_{\mu \pm}^{c}(x)+g f^{c b a} Q_{\mu \pm}^{b}(x) Q_{\nu \pm}^{a}(x), \quad F_{\mu \nu}^{c 2}\left[Q_{ \pm}\right]=F^{\mu \nu c}\left[Q_{ \pm}\right] F_{\mu \nu}^{c}\left[Q_{ \pm}\right] \\
& D\left[Q_{ \pm}\right]=i \gamma^{\mu} \partial_{\mu}+g T^{c} \gamma^{\mu} Q_{\mu \pm}^{c} .
\end{aligned}
$$

As mentioned above the Faddeev-Popov (F-P) determinant $\operatorname{det}\left(\frac{\delta\left(\partial_{\mu} Q_{ \pm}^{\mu b}\right)}{\delta \omega_{ \pm}^{c}}\right)$ in eq. (13) can be expressed in terms of path integral over the ghost fields [18] but we will directly work with the Faddeev-Popov (F-P) determinant $\operatorname{det}\left(\frac{\delta\left(\partial_{\mu} Q_{ \pm}^{\mu b}\right)}{\delta \omega_{ \pm}^{c}}\right)$ in this paper.

Note that the generating functional in non-equilibrium QCD in eq. (13) is generic from which the finite temperature QCD formalism and QCD in vacuum formalism can be obtained.

From eq. (13) we find that the non-perturbative gluon correlation function in non- 
equilibrium QCD is given by

$$
\begin{aligned}
& <\operatorname{in}\left|Q_{\mu r}^{a}\left(x_{1}\right) Q_{\nu s}^{b}\left(x_{2}\right)\right| i n>=\int\left[d Q_{+}\right]\left[d Q_{-}\right]\left[d \bar{\psi}_{+}\right]\left[d \bar{\psi}_{-}\right]\left[d \psi_{+}\right]\left[d \psi_{-}\right] Q_{\mu r}^{a}\left(x_{1}\right) Q_{\nu s}^{b}\left(x_{2}\right) \\
& \operatorname{det}\left(\frac{\delta \partial_{\mu} Q_{+}^{\mu c}}{\delta \omega_{+}^{d}}\right) \operatorname{det}\left(\frac{\delta \partial_{\mu} Q_{-}^{\mu c}}{\delta \omega_{-}^{d}}\right) \exp \left[i \int d ^ { 4 } x \left[-\frac{1}{4} F_{\mu \nu}^{c 2}\left[Q_{+}\right]+\frac{1}{4} F_{\mu \nu}^{c 2}\left[Q_{-}\right]-\frac{1}{2 \alpha}\left(\partial_{\mu} Q_{+}^{\mu c}\right)^{2}+\frac{1}{2 \alpha}\left(\partial_{\mu} Q_{-}^{\mu c}\right)^{2}\right.\right. \\
& \left.\left.+\bar{\psi}_{+} D\left[Q_{+}\right] \psi_{+}-\bar{\psi}_{-} D^{\prime}\left[Q_{-}\right] \psi_{-}\right]\right]<Q_{+}, \psi_{+}, \bar{\psi}_{+}, 0|\rho| 0, \bar{\psi}_{-}, \psi_{-}, Q_{-}>
\end{aligned}
$$

where $r, s=+,-$ are the closed-time path indices (" + "sign corresponds to positive time path and " - " sign corresponds to negative time path in the closed-time path formalism $[6,7])$.

\section{GAUGE NON-INVARIANT DEFINITION OF GLUON TO HADRON FRAG- MENTATION FUNCTION IN NON-EQUILIBRIUM QCD}

For simplicity, let us consider the scalar gluon in non-equilibrium first before considering the gluon in non-equilibrium QCD. For the application to RHIC and LHC high energy nuclear collisions it is useful to consider the light-cone coordinate system $x^{\mu}=\left(x^{+}, x^{-}, x_{T}\right)$. Note that at the initial time $x^{+}=x_{i n}^{+}=0$, the interaction picture coincides with the Heisenberg picture and Schrodinger picture. Hence at the initial time $x^{+}=x_{i n}^{+}=0$ we write the scalar field as

$$
\phi\left(x^{-}, x_{T}\right)=\frac{1}{(2 \pi)^{d-1}} \int \frac{d p^{+}}{\sqrt{2 p^{+}}} d^{d-2} p_{T}\left[e^{-i p^{+} x^{-}+i p_{T} \cdot x_{T}} a\left(p^{+}, p_{T}\right)+e^{i p^{+} x^{-}-i p_{T} \cdot x_{T}} a^{\dagger}\left(p^{+}, p_{T}\right)\right]
$$

where $a^{\dagger}(p)$ and $a(p)$ are the creation and the annihilation operators of the scalar gluon respectively. The commutation relations at the initial time are given by

$$
\begin{aligned}
& {\left[a\left(p^{+}, p_{T}\right), a^{\dagger}\left({p^{\prime}}^{+}, p_{T}^{\prime}\right)\right]_{x^{+}=0}=(2 \pi)^{d-1} \delta\left(p^{+}-p^{\prime+}\right) \delta^{(d-2)}\left(p_{T}-p_{T}^{\prime}\right)} \\
& {\left[a\left(p^{+}, p_{T}\right), a\left(p^{\prime+}, p_{T}^{\prime}\right)\right]_{x^{+}=0}=\left[a^{\dagger}\left(p^{+}, p_{T}\right), a^{\dagger}\left(p^{\prime+}, p_{T}^{\prime}\right)\right]_{x^{+}=0}=0 .}
\end{aligned}
$$

The non-equilibrium distribution function $f\left(p^{+}, p_{T}\right)$ of the fragmenting scalar gluon at initial time is given by

$$
<i n\left|a^{\dagger}\left(p^{+}, p_{T}\right) a\left(p^{+}, p_{T}^{\prime}\right)\right| i n>=f\left(p^{+}, p_{T}\right)(2 \pi)^{d-1} \delta\left(p^{+}-p^{++}\right) \delta^{(d-2)}\left(p_{T}-p_{T}^{\prime}\right)
$$

where we have assumed the space $\left(x^{-}\right.$and $\left.x_{T}\right)$ translational invariance at initial time $x^{+}=$

$x_{i n}^{+}=0$. The special case $f_{g}(\vec{p})=\frac{1}{e^{\frac{p_{0}}{T}}-1}$ corresponds to the finite temperature field theory in equilibrium. 
At the initial time $x^{+}=x_{i n}^{+}=0$ the scalar gluon state in the non-equilibrium quantum field theory is given by [10]

$$
\left|p^{+}, p_{T}>=a^{\dagger}\left(p^{+}, p_{T}\right)\right| \text { in }>
$$

where $a^{\dagger}(p)$ is the creation operator of the scalar gluon and $\mid i n>$ is the initial state of the non-equilibrium medium. By using eqs. (19), (18) and (17) we find, at initial time,

$$
\begin{aligned}
& <p^{+}, p_{T}\left|p^{+}, p_{T}^{\prime}>=<i n\right| a\left(p^{+}, p_{T}\right) a^{\dagger}\left(p^{+}, p_{T}^{\prime}\right) \mid i n> \\
& =(2 \pi)^{d-1} \delta\left(p^{+}-p^{\prime+}\right) \delta^{d-2}\left(p_{T}-p_{T}^{\prime}\right)\left[1+f\left(p^{+}, p_{T}\right)\right] .
\end{aligned}
$$

Consider the inclusive production of hadron $H$ created in the out-state $\mid H+X>$ from a scalar gluon in non-equilibrium in the initial state $\mid p>$ with the probability amplitude

$$
<H+X \mid p>
$$

where $X$ being other outgoing final state hadrons. The distribution $h_{p}(P)$ of the hadron $H$ with momentum $P$ from the parton of momentum $p$ in non-equilibrium can be found from the amplitude in eq. (21) by using

$$
\sum_{X}<p, p_{T}|H+X><H+X| p^{+^{\prime}}, p_{T}^{\prime}>=h_{p}(P)<p^{+}, p_{T} \mid p^{+^{\prime}}, p_{T}^{\prime}>
$$

We write the left hand side as

$$
\begin{aligned}
& \sum_{X}<p^{+}, p_{T}|H+X><H+X| p^{+^{\prime}}, p_{T}^{\prime}>=\sum_{X}<p^{+}, p_{T}\left|a_{H}^{\dagger}(P)\right| X><X\left|a_{H}(P)\right| p^{+^{\prime}}, p_{T}^{\prime}> \\
& =<p^{+}, p_{T}\left|a_{H}^{\dagger}(P) a_{H}(P)\right| p^{+^{\prime}}, p_{T}^{\prime}>.
\end{aligned}
$$

From eqs. (22), (23), (19) and (20) we find

$$
\begin{aligned}
& <i n\left|a\left(p^{+}, p_{T}\right) a_{H}^{\dagger}\left(P^{+}, P_{T}\right) a_{H}\left(P^{+}, P_{T}\right) a^{\dagger}\left(p^{\prime+}, p_{T}^{\prime}\right)\right| i n> \\
& =2 z(2 \pi)^{d-1} D_{H / a}\left(z, P_{T}\right)\left[1+f\left(p^{+}, p_{T}\right)\right] \delta\left(p^{+}-p^{\prime+}\right) \delta^{d-2}\left(p_{T}-p_{T}^{\prime}\right) .
\end{aligned}
$$

From eq. (16) we obtain

$$
\begin{aligned}
& (2 \pi)^{d-1}<i n\left|\phi\left(x^{-}, x_{T}\right) a_{H}^{\dagger}\left(P^{+}, P_{T}\right) a_{H}\left(P^{+}, P_{T}\right) \phi(0)\right| i n>=\frac{1}{(2 \pi)^{d-1}} \int \frac{d p^{+}}{\sqrt{2 p^{+}}} d^{d-2} p_{T} \int \frac{d p^{\prime+}}{\sqrt{2 p^{\prime}}} d^{d-2} p_{T}^{\prime} \\
& {\left[<i n\left|e^{-i p \cdot x} a\left(p^{+}, p_{T}\right) a_{H}^{\dagger}\left(P^{+}, P_{T}\right) a_{H}\left(P^{+}, P_{T}\right) a^{\dagger}\left(p^{\prime+}, p_{T}^{\prime}\right)\right| i n>\right]_{x^{+}=0}}
\end{aligned}
$$


Using this in eq. (24) we find the expression of the scalar gluon fragmentation function in non-equilibrium

$$
\begin{aligned}
& D_{H / a}\left(z, P_{T}\right)=\frac{p^{+}}{z\left[1+f\left(p^{+}, p_{T}\right)\right]} \int d x^{-} \frac{d^{d-2} x_{T}}{(2 \pi)^{d-1}} e^{i p^{+} x^{-}-i p_{T} \cdot x_{T}} \\
& <i n\left|\phi\left(x^{-}, x_{T}\right) a_{H}^{\dagger}\left(P^{+}, P_{T}\right) a_{H}\left(P^{+}, P_{T}\right) \phi(0)\right| i n>.
\end{aligned}
$$

By making a Lorentz transformation to the zero transverse momentum frame of the hadron [10] we find from eq. (26) that the scalar gluon fragmentation function in non-equilibrium is given by

$$
\begin{aligned}
& D_{H / a}\left(z, P_{T}\right)=\frac{p^{+}}{z\left[1+f\left(p^{+}, p_{T}\right)\right]} \int d x^{-} \frac{d^{d-2} x_{T}}{(2 \pi)^{d-1}} e^{i p^{+} x^{-}+i P_{T} \cdot x_{T} / z} \\
& <i n\left|\phi\left(x^{-}, x_{T}\right) a_{H}^{\dagger}\left(P^{+}, 0_{T}\right) a_{H}\left(P^{+}, 0_{T}\right) \phi(0)\right| i n>.
\end{aligned}
$$

In the above expression, $f\left(p^{+}, p_{T}\right)$ is the non-equilibrium distribution function of the fragmenting scalar gluon at initial time $x^{+}=x_{i n}^{+}=0$ and $\mid$ in $>$ is the initial state of the non-equilibrium medium in the Schwinger-Keldysh in - in closed-time path formalism.

In analogous to eq. (27) for the scalar gluon case, we find the gauge non-invariant definition of the gluon to hadron fragmentation function in non-equilibrium QCD is given by

$$
\begin{aligned}
& D_{H / g}\left(z, P_{T}\right)=\frac{p^{+}}{16 z\left[1+f_{g}\left(p^{+}, p_{T}\right)\right]} \int d x^{-} \frac{d^{d-2} x_{T}}{(2 \pi)^{d-1}} e^{i p^{+} x^{-}+i P_{T} \cdot x_{T} / z} \\
& <i n\left|Q^{\mu c}\left(x^{-}, x_{T}\right) a_{H}^{\dagger}\left(P^{+}, 0_{T}\right) a_{H}\left(P^{+}, 0_{T}\right) Q_{\mu}^{c}(0)\right| i n>
\end{aligned}
$$

where $f_{g}\left(p^{+}, p_{T}\right)$ is the non-equilibrium distribution function of the fragmenting gluon in QCD at initial time, $Q^{\mu c}(x)$ is the (quantum) gluon field in non-equilibrium QCD which fragments to hadron $H$ and $\mid$ in $>$ is the initial state of the non-equilibrium QCD medium in the Schwinger-Keldysh in - in closed-time path formalism.

Note that the transition from massless scalar gluon fragmentation function in eq. (27) to gauge non-invariant gluon fragmentation function in QCD in eq. (28) is achieved by the following procedure. The massless scalar gluon fragmentation function in quantum field theory in eq. (27) is proportional to the two-point scalar gluon correlation function of the type $<i n\left|\phi(x) a_{H}^{\dagger} a_{H} \phi(0)\right| i n>$. Note that, in quantum field theory, the two-point scalar gluon correlation function in scalar field theory is given by $<$ in $|\phi(x) \phi(0)| i n>$ and the two-point gluon correlation function in QCD is given by $<i n\left|Q_{\mu}^{a}(x) Q_{\nu}^{b}(0)\right|$ in $>$ where $\mu, \nu$ 
are Lorentz indices and $a, b$ are color indices of the gluon fields $Q_{\mu}^{a}(x)$ and $Q_{\nu}^{b}(0)$ in QCD. As mentioned above, in quantum field theory, the gluon fragmentation function is proportional to two-point gluon correlation function. Hence the gluon fragmentation function in QCD in eq. (28) is obtained from the massless scalar gluon fragmentation function in eq. (27) by replacing

$$
<i n\left|\phi(x) a_{H}^{\dagger} a_{H} \phi(0)\right| i n>\quad \rightarrow \quad<i n\left|Q_{\mu}^{a}(x) a_{H}^{\dagger} a_{H} Q^{\mu a}(0)\right| i n>
$$

Since the fragmentation function in QCD is a Lorentz scalar and color neutral quantity, the sum over Lorentz and color indices are taken in eq. (28). The additional factor $\frac{1}{16}$ in eq. (28) is supplied because the average over the spin and color $\left(\frac{1}{2 \times 8}\right)$ of the gluon in QCD is taken which is absent in the scalar gluon case.

\section{PROOF OF FACTORIZATION OF INFRARED DIVERGENCES IN NON- EQUILIBRIUM QCD}

Since the gluon to hadron fragmentation function in non-equilibrium QCD in eq. (28) is a non-perturbative quantity we will use path integral formulation of quantum field theory for this purpose [19, 20].

The generating functional in the background field method of QCD in non-equilibrium QCD in the path integral formulation is given by [8, 9, 12 15]

$$
\begin{aligned}
& Z\left[\rho, A, J_{+}, J_{-}, \eta_{+}, \eta_{-}, \bar{\eta}_{+}, \bar{\eta}_{-}\right]=\int\left[d Q_{+}\right]\left[d Q_{-}\right]\left[d \bar{\psi}_{+}\right]\left[d \bar{\psi}_{-}\right]\left[d \psi_{+}\right]\left[d \psi_{-}\right] \\
& \operatorname{det}\left(\frac{\delta G^{c}\left(Q_{+}\right)}{\delta \omega_{+}^{d}}\right) \times \operatorname{det}\left(\frac{\delta G^{c}\left(Q_{-}\right)}{\delta \omega_{-}^{d}}\right) \times \exp \left[i \int d ^ { 4 } x \left[-\frac{1}{4} F_{\mu \nu}^{c 2}\left[Q_{+}+A_{+}\right]+\frac{1}{4} F_{\mu \nu}^{c 2}\left[Q_{-}+A_{-}\right]\right.\right. \\
& -\frac{1}{2 \alpha}\left(G^{c}\left(Q_{+}\right)\right)^{2}+\frac{1}{2 \alpha}\left(G^{c}\left(Q_{-}\right)\right)^{2}+\bar{\psi}_{+} D\left[Q_{+}+A_{+}\right] \psi_{+} \\
& \left.\left.-\bar{\psi}_{-} D\left[Q_{-}+A_{-}\right] \psi_{-}+J_{+} \cdot Q_{+}-J_{-} \cdot Q_{-}+\bar{\eta}_{+} \cdot \psi_{+}-\bar{\eta}_{-} \cdot \psi_{-}+\bar{\psi}_{+} \cdot \eta_{+}-\bar{\psi}_{-} \cdot \eta_{-}\right]\right] \\
& <Q_{+}+A_{+}, \psi_{+}, \bar{\psi}_{+}, 0|\rho| 0, \bar{\psi}_{-}, \psi_{-}, Q_{-}+A_{-}>
\end{aligned}
$$

where the gauge fixing term is given by

$$
G^{c}\left(Q_{ \pm}\right)=\partial_{\mu} Q_{ \pm}^{\mu c}+g f^{c b a} A_{\mu \pm}^{b} Q_{ \pm}^{\mu a}=D_{\mu}\left[A_{ \pm}\right] Q_{ \pm}^{\mu c}
$$

which depends on the background field $A^{\mu c}(x)$ and

$$
F_{\mu \nu}^{c}\left[A_{ \pm}+Q_{ \pm}\right]=\partial_{\mu}\left[A_{\nu \pm}^{c}+Q_{\nu \pm}^{c}\right]-\partial_{\nu}\left[A_{\mu \pm}^{c}+Q_{\mu \pm}^{c}\right]+g f^{c b a}\left[A_{\mu \pm}^{b}+Q_{\mu \pm}^{b}\right]\left[A_{\nu \pm}^{a}+Q_{\nu \pm}^{a}\right]
$$


where $Q^{\mu c}$ is the quantum gluon field and $A^{\mu c}$ is the background field [12 14 .

From eq. (30) we find that the nonequilibrium-nonperturbative gluon correlation function in the background field method of QCD is given by

$$
\begin{aligned}
& <i n\left|Q_{\mu r}^{a}\left(x_{1}\right) Q_{\nu s}^{b}\left(x_{2}\right)\right| i n>_{A}=\int\left[d Q_{+}\right]\left[d Q_{-}\right]\left[d \bar{\psi}_{+}\right]\left[d \bar{\psi}_{-}\right]\left[d \psi_{+}\right]\left[d \psi_{-}\right] Q_{\mu r}^{a}\left(x_{1}\right) Q_{\nu s}^{b}\left(x_{2}\right) \\
& \operatorname{det}\left(\frac{\delta G^{c}\left(Q_{+}\right)}{\delta \omega_{+}^{d}}\right) \operatorname{det}\left(\frac{\delta G^{c}\left(Q_{-}\right)}{\delta \omega_{-}^{d}}\right) \exp \left[i \int d ^ { 4 } x \left[-\frac{1}{4} F_{\mu \nu}^{c 2}\left[Q_{+}+A_{+}\right]+\frac{1}{4} F_{\mu \nu}^{c 2}\left[Q_{-}+A_{-}\right]\right.\right. \\
& -\frac{1}{2 \alpha}\left(G^{c}\left(Q_{+}\right)\right)^{2}+\frac{1}{2 \alpha}\left(G^{c}\left(Q_{-}\right)\right)^{2}+\bar{\psi}_{+} D\left[Q_{+}+A_{+}\right] \psi_{+} \\
& \left.\left.-\bar{\psi}_{-} D\left[Q_{-}+A_{-}\right] \psi_{-}\right]\right]<Q_{+}+A_{+}, \psi_{+}, \bar{\psi}_{+}, 0|\rho| 0, \bar{\psi}_{-}, \psi_{-}, Q_{-}+A_{-}>.
\end{aligned}
$$

For a light-like quark attached to infinite number of gluons we find the eikonal factor [20]

$$
\begin{aligned}
& 1+g T^{c} \int \frac{d^{4} k}{(2 \pi)^{4}} \frac{l \cdot A^{c}(k)}{l \cdot k+i \epsilon}+g^{2} \int \frac{d^{4} k_{1}}{(2 \pi)^{4}} \frac{d^{4} k_{2}}{(2 \pi)^{4}} \frac{T^{c} l \cdot A^{c}\left(k_{1}\right) T^{b} l \cdot A^{b}\left(k_{2}\right)}{\left(l \cdot k_{1}+i \epsilon\right)\left(l \cdot\left(k_{1}+k_{2}\right)+i \epsilon\right)}+\ldots \\
& =\mathcal{P} \exp \left[i g \int_{0}^{\infty} d \lambda l \cdot A^{c}(l \lambda) T^{c}\right]
\end{aligned}
$$

which describes the infrared divergences arising from the infinite number of soft gluons exchange with the light-like quark of four-velocity $l^{\mu}$ where $\mathcal{P}$ is the path ordering and the gluon field $A^{\mu c}(x)$ and its Fourier transform $A^{\mu c}(k)$ are related by

$$
A^{\mu c}(x)=\int \frac{d^{4} k}{(2 \pi)^{4}} A^{\mu c}(k) e^{i k \cdot x} .
$$

The light-like quark traveling with light-like four-velocity $l^{\mu}$ produces SU(3) pure gauge field $A^{\mu c}(x)$ both in classical mechanics [21 23] and in quantum field theory [24] at all the timespace position $x^{\mu}$ except at the position $\vec{x}$ perpendicular to the direction of motion of the quark $(\vec{l} \cdot \vec{x}=0)$ at the time of closest approach $\left(x_{0}=0\right)$. When $A^{\mu c}(x)=A^{\mu c}(\lambda l)$ as in eq. (34) we find $\vec{l} \cdot \vec{x}=\lambda \vec{l} \cdot \vec{l}=\lambda \neq 0$ which implies that the light-like quark finds the gluon field $A^{\mu c}(x)$ in eq. (34) as the $\mathrm{SU}(3)$ pure gauge. The $\mathrm{SU}(3)$ pure gauge is given by

$$
T_{i j}^{a} A^{\mu a}(x)=\frac{1}{i g}\left[\left[\partial^{\mu} \Phi(x)\right] \Phi^{-1}(x)\right]_{i j}
$$

which gives the non-abelian gauge link [20]

$$
\Phi_{i j}(x)=\left[\mathcal{P} \exp \left[-i g \int_{0}^{\infty} d \lambda l \cdot A^{c}(x+l \lambda) T^{(A) c}\right]\right]_{i j}=\left[e^{i g T^{a} \omega^{a}(x)}\right]_{i j} .
$$

The gauge fixing term $\frac{1}{2 \alpha}\left(G^{a}(Q)\right)^{2}$ in eq. (30) [where $G^{a}(Q)$ is given by eq. (31)] is invariant for gauge transformation of $A_{\mu}^{a}$ :

$$
\delta A_{\mu}^{a}=g f^{a b c} A_{\mu}^{b} \omega^{c}+\partial_{\mu} \omega^{a}
$$


provided one also performs a homogeneous transformation of $Q_{\mu}^{a}[12,14]$ :

$$
\delta Q_{\mu}^{a}=g f^{a b c} Q_{\mu}^{b} \omega^{c}
$$

By changing the integration variable $Q \rightarrow Q-A$ in the right hand side of eq. (33) we find

$$
\begin{aligned}
& <i n\left|Q_{\mu r}^{a}\left(x_{1}\right) Q_{\nu s}^{b}\left(x_{2}\right)\right| i n>_{A}=\int\left[d Q_{+}\right]\left[d Q_{-}\right]\left[d \bar{\psi}_{+}\right]\left[d \bar{\psi}_{-}\right]\left[d \psi_{+}\right]\left[d \psi_{-}\right]\left[Q_{\mu r}^{a}\left(x_{1}\right)-A_{\mu r}^{a}\left(x_{1}\right)\right] \\
& {\left[Q_{\nu s}^{b}\left(x_{2}\right)-A_{\nu s}^{b}\left(x_{2}\right] \operatorname{det}\left(\frac{\delta G_{f}^{c}\left(Q_{+}\right)}{\delta \omega_{+}^{d}}\right) \operatorname{det}\left(\frac{\delta G_{f}^{c}\left(Q_{-}\right)}{\delta \omega_{-}^{d}}\right) \exp \left[i \int d ^ { 4 } x \left[-\frac{1}{4} F_{\mu \nu}^{c 2}\left[Q_{+}\right]+\frac{1}{4} F_{\mu \nu}^{c 2}\left[Q_{-}\right]\right.\right.\right.} \\
& \left.\left.-\frac{1}{2 \alpha}\left(G_{f}^{c}\left(Q_{+}\right)\right)^{2}+\frac{1}{2 \alpha}\left(G_{f}^{c}\left(Q_{-}\right)\right)^{2}+\bar{\psi}_{+} D\left[Q_{+}\right] \psi_{+}-\bar{\psi}_{-} D\left[Q_{-}\right] \psi_{-}\right]\right]<Q_{+}, \psi_{+}, \bar{\psi}_{+}, 0|\rho| 0, \bar{\psi}_{-}, \psi_{-}, Q_{-}>
\end{aligned}
$$

where from eq. (31) we find

$$
G_{f}^{c}\left(Q_{ \pm}\right)=\partial_{\mu} Q_{ \pm}^{\mu a}+g f^{c b a} A_{\mu \pm}^{b} Q_{ \pm}^{\mu a}-\partial_{\mu} A_{ \pm}^{\mu c}=D_{\mu}\left[A_{ \pm}\right] Q_{ \pm}^{\mu c}-\partial_{\mu} A_{ \pm}^{\mu c}
$$

and from eq. (39) [by using eq. (38)] we find

$$
\delta Q_{\mu \pm}^{c}=-g f^{c b a} \omega_{ \pm}^{b} Q_{\mu \pm}^{a}+\partial_{\mu} \omega_{ \pm}^{c}
$$

Changing the integration variable from unprimed variable to primed variable we find from eq. (40)

$$
\begin{aligned}
& <i n\left|Q_{\mu r}^{a}\left(x_{1}\right) Q_{\nu s}^{b}\left(x_{2}\right)\right| \text { in }>_{A}=\int\left[d Q_{+}^{\prime}\right]\left[d Q_{-}^{\prime}\right]\left[d \bar{\psi}_{+}^{\prime}\right]\left[d \bar{\psi}_{-}^{\prime}\right]\left[d \psi_{+}^{\prime}\right]\left[d \psi_{-}^{\prime}\right]\left[Q_{\mu r}^{a}\left(x_{1}\right)-A_{\mu r}^{a}\left(x_{1}\right)\right] \\
& {\left[Q_{\nu s}^{\prime b}\left(x_{2}\right)-A_{\nu s}^{b}\left(x_{2}\right] \times \operatorname{det}\left(\frac{\delta G_{f}^{c}\left(Q_{+}^{\prime}\right)}{\delta \omega_{+}^{d}}\right) \operatorname{det}\left(\frac{\delta G_{f}^{c}\left(Q_{-}^{\prime}\right)}{\delta \omega_{-}^{d}}\right) \exp \left[i \int d ^ { 4 } x \left[-\frac{1}{4} F_{\mu \nu}^{c 2}\left[Q_{+}^{\prime}\right]+\frac{1}{4} F_{\mu \nu}^{c 2}\left[Q_{-}^{\prime}\right]\right.\right.\right.} \\
& \left.\left.-\frac{1}{2 \alpha}\left(G_{f}^{c}\left(Q_{+}^{\prime}\right)\right)^{2}+\frac{1}{2 \alpha}\left(G_{f}^{c}\left(Q_{-}^{\prime}\right)\right)^{2}+\bar{\psi}_{+}^{\prime} D\left[Q_{+}^{\prime}\right] \psi_{+}^{\prime}-\bar{\psi}_{-}^{\prime} D\left[Q_{-}^{\prime}\right] \psi_{-}^{\prime}\right]\right]<Q_{+}^{\prime}, \psi_{+}^{\prime}, \bar{\psi}_{+}^{\prime}, 0|\rho| 0, \bar{\psi}_{-}^{\prime}, \psi_{-}^{\prime}, Q_{-}^{\prime}>
\end{aligned}
$$

This is because a change of integration variable from unprimed variable to primed variable does not change the value of the integration.

The equation

$$
Q_{\mu \pm}^{c c}(x)=Q_{\mu \pm}^{a}(x)+g f^{c b a} \omega_{ \pm}^{a}(x) Q_{\mu \pm}^{b}(x)+\partial_{\mu} \omega_{ \pm}^{c}(x)
$$

in eq. (42) is valid for infinitesimal transformation $(\omega<<1)$ which is obtained from the finite equation

$$
T^{c} Q_{\mu \pm}^{\prime c}(x)=U_{ \pm}(x) T^{c} Q_{\mu \pm}^{c}(x) U_{ \pm}^{-1}(x)+\frac{1}{i g}\left[\partial_{\mu} U_{ \pm}(x)\right] U_{ \pm}^{-1}(x), \quad U_{ \pm}(x)=e^{i g T^{c} \omega_{ \pm}^{c}(x)}
$$


Simplifying infinite numbers of non-commuting terms we find

$$
Q_{\mu \pm}^{\prime c}(x)=\left[e^{g M_{ \pm}(x)}\right]_{c b} Q_{\mu \pm}^{b}(x)+\left[\frac{e^{g M_{ \pm}(x)}-1}{g M_{ \pm}(x)}\right]_{c b}\left[\partial_{\mu} \omega_{ \pm}^{b}(x)\right]
$$

where

$$
M_{ \pm}^{c b}(x)=f^{c b a} \omega_{ \pm}^{a}(x)
$$

The fermion field transforms as

$$
\psi_{ \pm}^{\prime}(x)=e^{i g T^{c} \omega_{ \pm}^{c}(x)} \psi_{ \pm}(x)
$$

Under the finite transformation, using eqs. (46), (48) and [20] we find

$$
\begin{aligned}
& {\left[d Q_{ \pm}^{\prime}\right]=\left[d Q_{ \pm}\right], \quad\left[d \bar{\psi}_{ \pm}^{\prime}\right]\left[d \psi_{ \pm}^{\prime}\right]=\left[d \bar{\psi}_{ \pm}\right]\left[d \psi_{ \pm}\right], \quad F_{\mu \nu}^{a 2}\left[Q_{ \pm}^{\prime}\right]=F_{\mu \nu}^{a 2}\left[Q_{ \pm}\right], \quad\left(G_{f}^{c}\left(Q_{ \pm}^{\prime}\right)\right)^{2}=\left(\partial_{\mu} Q_{ \pm}^{\mu c}(x)\right)^{2}} \\
& \bar{\psi}_{ \pm}^{\prime}\left[i \gamma^{\mu} \partial_{\mu}-m+g T^{c} \gamma^{\mu} Q_{\mu \pm}^{\prime c}\right] \psi_{ \pm}^{\prime}=\bar{\psi}_{ \pm}\left[i \gamma^{\mu} \partial_{\mu}-m+g T^{c} \gamma^{\mu} Q_{\mu \pm}^{c}\right] \psi_{ \pm} \\
& \operatorname{det}\left[\frac{\delta G_{f}^{c}\left(Q_{ \pm}^{\prime}\right)}{\delta \omega_{ \pm}^{d}}\right]=\operatorname{det}\left[\frac{\delta\left(\partial_{\mu} Q_{ \pm}^{\mu c}(x)\right)}{\delta \omega_{ \pm}^{d}}\right], \quad Q_{\mu \pm}^{\prime c}(x)-A_{\mu \pm}^{c}(x)=\left[e^{g M_{ \pm}(x)}\right]_{c b} Q_{\mu \pm}^{b}(x) .
\end{aligned}
$$

Since we are working in the frozen ghost formalism at initial time the < $Q_{+}, \psi_{+}, \bar{\psi}_{+}, 0|\rho| 0, \bar{\psi}_{-}, \psi_{-}, Q_{-}>$corresponding to initial density of states in eq. (15) is gauge invariant by definition. Hence from eqs. (46) and (48) we find [11]

$$
<Q_{+}^{\prime}, \psi_{+}^{\prime}, \bar{\psi}_{+}^{\prime}, 0|\rho| 0, \bar{\psi}_{-}^{\prime}, \psi_{-}^{\prime}, Q_{-}^{\prime}>=<Q_{+}, \psi_{+}, \bar{\psi}_{+}, 0|\rho| 0, \bar{\psi}_{-}, \psi_{-}, Q_{-}>
$$

Using eqs. (49) and (50) in eq. (43) we find

$$
\begin{aligned}
& <i n\left|Q_{\mu r}^{a}\left(x_{1}\right) Q_{\nu s}^{b}\left(x_{2}\right)\right| i n>_{A}=\int\left[d Q_{+}\right]\left[d Q_{-}\right]\left[d \bar{\psi}_{+}\right]\left[d \bar{\psi}_{-}\right]\left[d \psi_{+}\right]\left[d \psi_{-}\right]\left[e^{g M_{r}\left(x_{1}\right)}\right]_{a c} Q_{\mu r}^{c}\left(x_{1}\right) \\
& {\left[e^{g M_{s}\left(x_{2}\right)}\right]_{b d} Q_{\nu s}^{d}\left(x_{2}\right) \operatorname{det}\left(\frac{\delta \partial_{\mu} Q_{+}^{\mu c}}{\delta \omega_{+}^{d}}\right) \operatorname{det}\left(\frac{\delta \partial_{\mu} Q_{-}^{\mu c}}{\delta \omega_{-}^{d}}\right) \exp \left[i \int d ^ { 4 } x \left[-\frac{1}{4} F_{\mu \nu}^{c 2}\left[Q_{+}\right]+\frac{1}{4} F_{\mu \nu}^{c 2}\left[Q_{-}\right]\right.\right.} \\
& \left.\left.-\frac{1}{2 \alpha}\left(\partial_{\mu} Q_{+}^{\mu c}\right)^{2}+\frac{1}{2 \alpha}\left(\partial_{\mu} Q_{-}^{\mu c}\right)^{2}+\bar{\psi}_{+} D\left[Q_{+}\right] \psi_{+}-\bar{\psi}_{-} D\left[Q_{-}\right] \psi_{-}\right]\right]<Q_{+}, \psi_{+}, \bar{\psi}_{+}, 0|\rho| 0, \bar{\psi}_{-}, \psi_{-}, Q_{-}>
\end{aligned}
$$

Using the similar technique as above we find

$$
\begin{aligned}
& <\operatorname{in|}\left|\left[e^{g M_{r}\left(x_{1}\right)}\right]_{a c} Q_{\mu r}^{c}\left(x_{1}\right)\left[e^{g M_{s}\left(x_{2}\right)}\right]_{b d} Q_{\nu s}^{d}\left(x_{2}\right)\right| i n>_{A}=\int\left[d Q_{+}\right]\left[d Q_{-}\right]\left[d \bar{\psi}_{+}\right]\left[d \bar{\psi}_{-}\right]\left[d \psi_{+}\right]\left[d \psi_{-}\right] \\
& Q_{\mu r}^{a}\left(x_{1}\right) Q_{\nu s}^{b}\left(x_{2}\right) \operatorname{det}\left(\frac{\delta \partial_{\mu} Q_{+}^{\mu c}}{\delta \omega_{+}^{d}}\right) \operatorname{det}\left(\frac{\delta \partial_{\mu} Q_{-}^{\mu c}}{\delta \omega_{-}^{d}}\right) \exp \left[i \int d ^ { 4 } x \left[-\frac{1}{4} F_{\mu \nu}^{c 2}\left[Q_{+}\right]+\frac{1}{4} F_{\mu \nu}^{c 2}\left[Q_{-}\right]\right.\right. \\
& \left.\left.-\frac{1}{2 \alpha}\left(\partial_{\mu} Q_{+}^{\mu c}\right)^{2}+\frac{1}{2 \alpha}\left(\partial_{\mu} Q_{-}^{\mu c}\right)^{2}+\bar{\psi}_{+} D\left[Q_{+}\right] \psi_{+}-\bar{\psi}_{-} D\left[Q_{-}\right] \psi_{-}\right]\right]<Q_{+}, \psi_{+}, \bar{\psi}_{+}, 0|\rho| 0, \bar{\psi}_{-}, \psi_{-}, Q_{-}>
\end{aligned}
$$


where $M_{r}^{b c}(x)$ is given by eq. (47). From eqs. (15) and (152) we find

$$
<i n\left|Q_{\mu r}^{a}\left(x_{1}\right) Q_{\nu s}^{b}\left(x_{2}\right)\right| i n>=<i n\left|\left[e^{g M_{r}\left(x_{1}\right)}\right]_{a c} Q_{\mu r}^{c}\left(x_{1}\right)\left[e^{g M_{s}\left(x_{2}\right)}\right]_{b d} Q_{\nu s}^{d}\left(x_{2}\right)\right| i n>_{A}
$$

which is valid in covariant gauge, in light-cone gauge, in general axial gauges, in general noncovariant gauges and in general Coulomb gauge etc. [20]. Note that the creation operator $a^{\dagger}$ and annihilation operator $a$ of the quark are related to the quark field via the equation [25]

$$
\psi(x)=\sum_{\text {spin }} \sum_{p} \sqrt{\frac{m}{V E_{p}}}\left[a(p) u(p) e^{-i p \cdot x}+a^{\dagger}(p) v(p) e^{i p \cdot x}\right]
$$

where color indices are suppressed. Hence one finds that the quark field $\psi(x)$ depends on the the creation (annihilation) operator $a^{\dagger}(a)$ of the quark but is independent of the creation (annihilation) operator $a_{H}^{\dagger}\left(a_{H}\right)$ of the hadron. Similarly the gluon field $Q^{\mu a}(x)$ is

also independent of the creation (annihilation) operator $a_{H}^{\dagger}\left(a_{H}\right)$ of the hadron. Since $a_{H}^{\dagger} a_{H}$ is independent of $\psi(x)$ and $Q^{\mu a}(x)$ one can perform exactly the similar steps of the path integral calculation as above to find from eq. (53) that

$$
<i n\left|Q_{\mu r}^{a}\left(x_{1}\right) a_{H}^{\dagger} a_{H} Q_{\nu s}^{b}\left(x_{2}\right)\right| i n>=<i n\left|\Phi_{a c r}\left(x_{1}\right) Q_{\mu r}^{c}\left(x_{1}\right) a_{H}^{\dagger} a_{H} \Phi_{b d s}\left(x_{2}\right) Q_{\nu s}^{d}\left(x_{2}\right)\right| i n>_{A}
$$

which proves factorization of soft (infrared) divergences at all order in coupling constant in non-equilibrium QCD where [see eqs. (47) and (37)]

$$
\Phi_{b c r}(x)=\left[\mathcal{P} \exp \left[-i g \int_{0}^{\infty} d \lambda l \cdot A_{r}^{a}(x+l \lambda) T^{(A) a}\right]\right]_{b c}, \quad\left(T^{(A) a}\right)_{b c}=-i f^{a b c}
$$

is the non-abelian gauge link or non-abelian phase in the adjoint representation of $\mathrm{SU}(3), l^{\mu}$ is the light-like four velocity and $r=+,-$ corresponds to the upper and lower time branches in the closed-time path formalism [6, 7] and $a, b, c=1,2, \ldots, 8$ are color indices.

\section{GAUGE INVARIANT DEFINITION OF GLUON TO HADRON FRAGMEN- TATION FUNCTION IN NON-EQUILIBRIUM QCD}

The definition of the gluon to hadron fragmentation function in non-equilibrium QCD in eq. (28) is not gauge invariant. To make it gauge invariant and consistent with factorization 
of infrared divergences we need to incorporate Wilson lines. The non-abelian gauge link in the adjoint representation of $\mathrm{SU}(3)$ is given by the path ordered exponential, see eq. (56),

$$
\Phi_{a b}(x)=\left[\mathcal{P} \exp \left[-i g \int_{0}^{\infty} d \lambda l \cdot A^{c}(x+l \lambda) T^{(A) c}\right]\right]_{a b}, \quad\left(T^{(A) c}\right)_{a b}=-i f^{a b c}
$$

which under non-abelian gauge transformation, as given by eq. (3), transforms as

$$
\Phi_{a b}^{\prime}(x)=U_{a c}(x) \Phi_{c b}(x)
$$

where

$$
U_{a b}(x)=\left[e^{i g T^{(A) c} \omega^{c}(x)}\right]_{a b}
$$

and from eq. (4) we find

$$
Q_{\mu r}^{\prime a}(x)=U_{a b}(x) Q_{\mu r}^{b}(x)
$$

Hence by extending the path integral formulation of quark fragmentation function in non-equilibrium QCD [11] to gluon fragmentation function in non-equilibrium QCD we find from eqs. (55) $,(56),(28),(15),(58)$ and (160) that the gauge invariant definition of the gluon to hadron fragmentation function in non-equilibrium QCD which is obtained from the single

gluon in-state $\mid g>$ and is consistent with factorization theorem in non-equilibrium QCD is given by

$$
\begin{aligned}
& D_{H / g}\left(z, P_{T}\right)=\frac{k^{+}}{16 z\left[1+f_{g}\left(k^{+}, k_{T}\right)\right]} \int d x^{-} \frac{d^{d-2} x_{T}}{(2 \pi)^{d-1}} e^{i k^{+} x^{-}+i P_{T} \cdot x_{T} / z} \\
& <i n\left|Q^{\mu a}\left(x^{-}, x_{T}\right) \Phi\left[x^{-}, x_{T}\right] a_{H}^{\dagger}\left(P^{+}, 0_{T}\right) a_{H}\left(P^{+}, 0_{T}\right) \Phi[0] Q_{\mu}^{a}(0)\right| i n>
\end{aligned}
$$

which reproduces eq. (11) which is valid in covariant gauge, in light-cone gauge, in general axial gauges, in general non-covariant gauges and in general Coulomb gauge etc. where the path ordered exponential $\Phi_{a b}[x]$ is given by eq. (2) .

This completes the derivation of gluon to hadron fragmentation function in nonequilibrium QCD.

\section{CONCLUSIONS}

Theoretical understanding of the observed jet quenching measurements at RHIC and LHC is challenging in QCD because it requires understanding of parton to hadron fragmentation function in non-equilibrium QCD. In this paper, by using closed-time path integral 
formalism, we have derived the gauge invariant definition of the gluon to hadron fragmentation function in non-equilibrium QCD which is consistent with factorization theorem in non-equilibrium QCD from first principles.

[1] T. Matsui and H. Satz, Phys. Lett. B178 (1986) 416.

[2] L. D. McLerran and T. Toimela, Phys. Rev. D31 (1985) 545.

[3] P. Koch, B. Muller and J. Rafelski, Phys. Rept. 142 (1986) 167.

[4] M. Gyulassy and X-N. Wang, Nucl.Phys. B420 (1994) 583; M. Gyulassy and X. N. Wang, Nucl. Phys. B420 (1994) 307; M. Gyulassy et al., In *Hwa, R.C. (ed.) et al.: Quark gluon plasma* 123-191, nucl-th/0302077.

[5] M. Panero, K. Rummukainen and A. Schaefer, Phys. Rev. Lett. 112 (2014) 162001, arXiv:1307.5850 [hep-ph].

[6] J. Schwinger, J. Math. Phys. 2 (1961) 407.

[7] L. V. Keldysh, JETP 20 (1965) 1018.

[8] F. Cooper, C-W. Kao and G. C. Nayak, Phys. Rev. D 66 (2002) 114016.

[9] C-W. Kao, G. C. Nayak and W. Greiner, Phys. Rev. D 66 (2002) 034017.

[10] G. C Nayak, Eur. Phys. J. C59 (2009) 891, arXiv:0808.1288 [hep-ph].

[11] G. C. Nayak, Annals Phys. 325 (2010) 682.

[12] L. F. Abbott, Nucl. Phys. B185 (1981) 189.

[13] G. 't Hooft, Nucl. Phys. B62 (1973) 444.

[14] H. Klueberg-Stern and J. B. Zuber, Phys. Rev. D12 (1975) 482.

[15] H. Klueberg-Stern and J. B. Zuber, Phys. Rev. D12 (1975) 3159.

[16] V. N. Gribov and L. N. Lipatov, Sov. J. Nucl. Phys. 15 (1972) 438; L. N. Lipatov, Sov. J. Nucl. Phys. 20 (1975) 94; G. Altarelli and G. Parisi, Nucl. Phys. B126 (1977) 298; Yu. L. Dokshitzer, Sov. Phys. J. Exp. Theor. Phys. 46 (1977) 641.

[17] G. C. Nayak, Phys. Part. Nucl. 43 (2012) 742, arXiv:0905.3922 [hep-ph].

[18] See for example, T. Muta, Foundations of Quantum Chromodynamics, World Scientific lecture notes in physics-Vol. 5.

[19] R. Tucci, Phys. Rev. D32 (1985) 945.

[20] G. C. Nayak, Phys. Part. Nucl. Lett. 13 (2016) 417, arXiv:1506.07146 [hep-ph]. 
[21] J. C. Collins, D. E. Soper and G. Sterman, Nucl. Phys. B261 (1985) 104.

[22] G. C. Nayak, JHEP1303(2013)001.

[23] G. C. Nayak, Eur. Phys. J. C73(2013)2442.

[24] G. C. Nayak, arXiv:1509.05303 [hep-ph].

[25] F. Mandl and G. Shaw, Quantum Field Theory, John Wiley and Sons, 1984. 